\title{
Application of Triple Features Theory to the Analysis of Half-Tone Images and Colored Textures. Feature Construction by Virtue of Stochastic Geometry and Functional Analysis
}

\author{
Nikolay Fedotov ${ }^{1}$, Sergey Romanov ${ }^{1} \&$ Daria Goldueva ${ }^{1}$ \\ ${ }^{1}$ Penza State University, Russian Federation \\ Correspondence: Nikolay Fedotov, Penza State University, Russian Federation. E-mail: nikolayfedotov@mail.ru
}

Received: May 27, 2013 Accepted: June 29, 2013 Online Published: August 8, 2013

doi:10.5539/cis.v6n4p17 URL: http://dx.doi.org/10.5539/cis.v6n4p17

\begin{abstract}
The existing methods of half-tone or color image recognition generally presuppose a prior simplification of the object to analyze. Such a simplification normally involves image binarization which may result in a loss of essential elements of information on the object. The paper proposes a new approach towards half-tone images and colored textures analysis and recognition by virtue of stochastic geometry and functional analysis. The method makes it possible to form both the recognition features to typify image geometric singularities, and the recognition features to reflect image brightness and color characteristics. According to the method suggested, recognition features can be generated in abundance - thousands of them - in an unattended mode, which provides for a most reliable image recognition. Moreover, the resulting features prove invariant both to a group of motions and to linear deformations, which is the key to the better part of image recognition problems.
\end{abstract}

Keywords: image recognition, half-tone image, color texture, trace-transformation, triple feature, stochastic geometry

\section{Introduction}

Most of the present-day technical devices of digital image representation rely on geometric singularities of images which makes them unfit to analyze half-tone and color images. Binarization of such images results in a loss of some essential background information, which impairs accuracy of their recognition. The method of image analysis and recognition put forward in the paper is based on stochastic geometry and functional analysis (Федотов, 1990, 2009; Kendall, Wilfrid, Molchanov, \& Ilya, 2010). It allows gaining both geometric features for recognition, and some extra features which reflect image brightness singularities. Usages of such features enhance the recognition system accuracy, robustness and versatility. The system becomes applicable to analyze binary, half-tone, and colored pictures.

The approach developed rests upon the mathematical apparatus of stochastic geometry and functional analysis. The key element to the method is a new geometric trace-transform concerned with the scanning of the object to be analyzed, along complex trajectories, introduced in (Федотов, 1990), and explored in (Федотов, 1990; Kadyrov, Saveleva, \& Fedotov, 1994; Fedotov \& Kadyrov, 1995; Fedotov \& Shulga, 2000; Kadyrov \& Fedotov, 1995), and the papers to succeed. In special cases, the trace-transform can be reduced to Radon, Radon Fourier, Ho, or Radon- Ho transform, but it is not equivalent to them (Федотов, 2009). Trace-transform presents a convenient tool for the recognition of moving objects. It can provide a basis for a new class of recognition features, which allows to achieve recognition, either invariant, or sensitive to a group of motions and to scaling (Kadyrov \& Fedotov, 1995). With the features to arrange sensitive recognition, one can further specify parameters of the transformation the initial object has undergone (Kadyrov \& Fedotov, 1995). Motions studied by means of trace-transform, have been discussed in (Kadyrov, Saveleva, \& Fedotov, 1994; Fedotov \& Shulga, 2000). In (Федотов, Кольчугин, Смолькин, Моисеев, \& Романов, 2008), a dual mapping was introduced, which, in combination with the trace-transform and triple features, lets preprocess images via nonlinear filtration before features can be constructed. Here, polygonal approximation, selecting convex hull, noise reduction, etc. can be applied (Федотов, Кольчугин, Смолькин, Моисеев, \& Романов, 2008; Vidal \& Amigo, 2012). Trace-transforms and the theory of triple features can be used to effectively solve a wide range of practical problems of images recognition and analysis in various fields of human activity. Application of trace-transform 
and the theory of triple features to detect welding defects were developed in (Федотов \& Никифорова, 2002). Using the above apparatus for biometric identification and recognizing human faces, was discussed in (Fedotov, Shulga, \& Roy, 2004; Shin, Cha, Kim, Cho, Klette, \& Young, 2008; Fooprateepsiri \& Kurutach, 2010). Analysis and recognition of biological nanoobjects can be found in (Федотов, 2009). That very book contains analysis of triple features construction precision. Fedotov, Shulga, Kol'chugin, Smol'kin and Romanov (2007) present the results of the ultrasonic analysis of images in medicine. Hence, the approach suggested has found a wide application.

The present paper considers development of the triple feature theory to the analysis of half-tone images and colored textures.

\section{Trace-Transform}

According to the method suggested, the first stage to form recognition features is a geometric trace-transform which assumes image scanning along complex trajectories, introduced in (Федотов, 1990). Applied researches generally focus on scanning images with a composition of discrete lattices composed of parallel lines. Other scanning trajectories are studied in (Федотов, 1990). Image $F(x, y)$ (Figure 1) is being scanned with a lattice of parallel lines spaced $\Delta \rho$ apart.

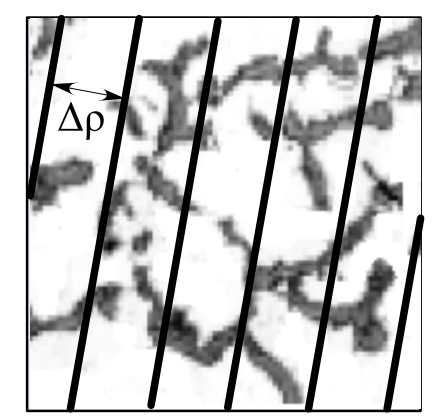

Figure 1. Scanning an image with a lattice of parallel lines

For binary images, each point $t$ of the scanning line $l(\theta, \rho, t)$ are assigned with a number belonging to the set $\{0$, $1\}$, according to the rule as follows:

$$
\forall t \in l(\theta, \rho, t) f(\theta, \rho, t)= \begin{cases}1 ; & t \in F \cap l, \\ 0 ; & t \notin F \cap l .\end{cases}
$$

By virtue of T-functional, we obtain a certain characteristics $g$ of the scanning line $l(\theta, \rho)$ and the image $F$ relative position, i.e.

$$
g(\theta, \rho)=\mathrm{T}(F \cap l(\theta, \rho))=\mathrm{T} f(\theta, \rho, t)
$$

The number of the line-and-image cross points, or the cross-point nhood properties, etc. can serve as such a characteristics.

Then, for the new angle value, which has got a discrete increment $\Delta \theta$, the image scanning is to be performed with a lattice of $\Delta \rho$-spaced lines.

The total of characteristics $g\left(\theta_{j}, \rho_{i}\right)$ of all possible scanning lines $l\left(\theta_{j}, \rho_{i}\right)$ and the image $F(x, y)$ relative positions, make a Trace-matrix (Figure 2).

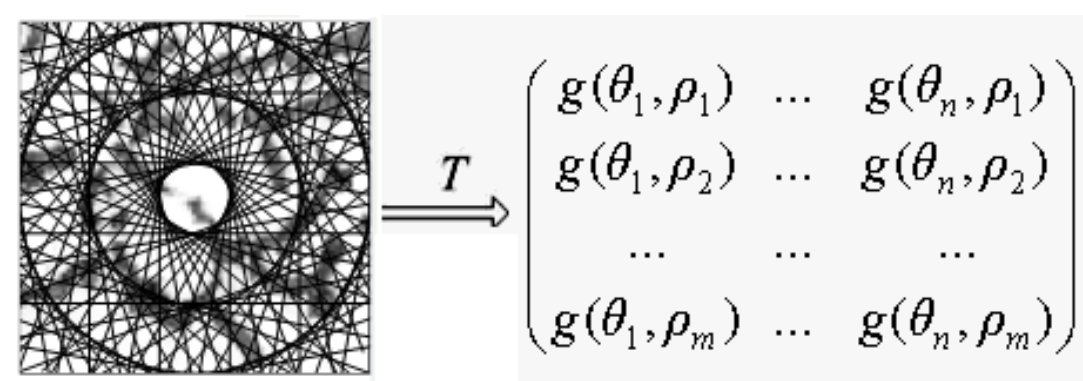

Figure 2. Creation of a trace-matrix 
In such a manner, the initial image $F(x, y)$ could be put into correspondence with a new image $\Phi(\theta, \rho)$, the colour (or brightness) in every point $\left(\theta_{j}, \rho_{i}\right)$ of the latter image being defined as $g\left(\theta_{j}, \rho_{i}\right)$. The image obtained is the Trace-transform of the initial image.

Figure 3 exemplifies an image and the corresponding Trace-transform.

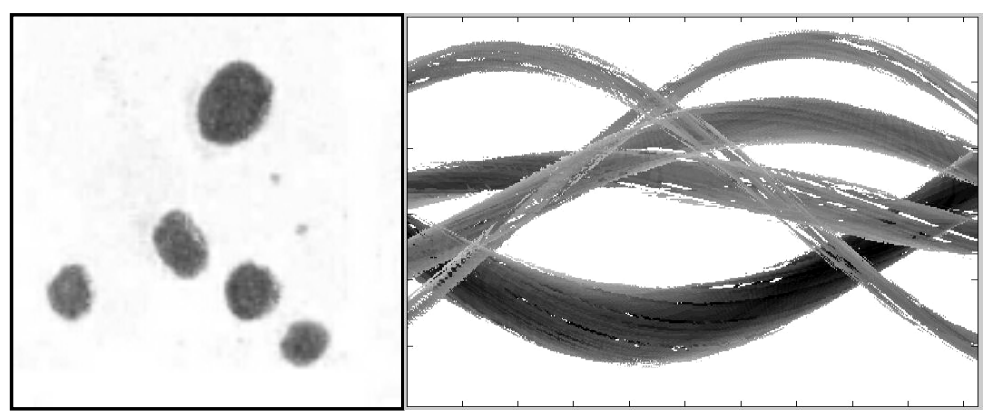

a) b)

Figure 3. a) Initial image; b) its trace-transform

The transformation, which translates the initial image into a Trace-transform, is a Trace-transformation.

Trace-transformation offers an efficient instrument to study motions of the objects to be recognized, and of their scale conversion. It can be accounted for by the fact that a trace-transform holds information on the initial object, i.e. the type of the trace-matrix stays unchanged under the action of a group of motion (rotation, translation), and linear deformations, each of the transformations introducing a peculiar component when creating a trace-transform. More specifically, when turning the initial image to a certain angle $\alpha$, trace-matrix values stay unchanged, its columns being shifted nonarithmetically along the abscissa $\mathrm{O} \theta$ to the angular displacement value $\alpha$. When the initial image is translated by a certain vector, trace-matrix values stay unchanged, the numbers in the columns shifting up or down. For the homothety with the coefficient $k>1$, i.e. when the initial image has been increased, the trace-matrix range $\rho$ is going to become $k$ times extended; when the image has been decreased $(k<1)$, the range $\rho$ is going to be contracted.

\section{Triple Features}

Our theory supposes that a trace-matrix having been completed, feature formation is to go on as follows.

Each column of the trace-matrix down the line, is to be applied with functional $\mathrm{P}$, which depends on the parameter $\rho$. Under the effect of the latter, each column of the trace-matrix rearranges to a real number. Hence, the application of the diametric functional to the trace-matrix, in the discrete variation, results in a vector, its $i$-th component being functional P meaning for the elements of the trace-matrix $i$-th column. Applying diametric functional to a trace-transform results in a $2 \pi$-periodic curve, which depends upon the parameter $\theta, h(\theta)=\mathrm{P}(g(\theta$, $\rho)$ ). Functional $\mathrm{P}$ per se has been called diametric for the parameter $\rho$ takes its largest values at the image diagonal points, corresponding to the diameter of the recognition system retina.

The last stage of feature formation is related to functional $\Theta$, which depends on the parameter $\theta$. Functional $\Theta$ assigns the set of the vector components, (or the set of points on the curve $h(\theta)$ ), which has been obtained a stage before, with a certain real number equal to the triple-feature meaning.

We call functional $\Theta$ circular, for the curve domain is $h(\theta) 2 \pi(0 \leq \theta \leq 2 \pi)$.

As the result, the recognition features suggested, having a structure composed of three functionals, were called triple features:

$$
\Pi(F)=\Theta \circ P \circ T(F \cap l(\rho, \theta))
$$

where $\mathrm{T}$ is a trace-functional, related to the natural coordinate $\mathrm{t}$ of the scanning line $l(\rho, \theta)$; $\mathrm{P}$ is a diametric functional, and $\Theta-$ a circular one, related to the scanning line normal coordinates, $\rho$ and $\theta$, correspondingly. Due to the trinary structure of triple features, one could automatically, using computer, generate a wide variety (some tens of thousands) of them. Leaning on a great number of features helps enhance recognition fidelity and affords an opportunity to speak about a different understanding of images (Fedotov \& Shulga, 2000; Fedotov \& Shulga, 2004). 
Functionals T, P, $\Theta$ are chosen from different fields of mathematics, such as theory of chances, mathematical statistics, fractal theory, stochastic geometry, etc. Hence, triple features keep traces of the relevant mathematical fields' geneses, which accounts for the flexibility and versatility of the recognition algorithms based on them (Fedotov \& Shulga, 2000). Particularly, if functionals are properly chosen, one could obtain features invariant to a group of motion and dilatation, which is of vital importance to half-toned and chromatic images recognition.

Let us exemplify a triple feature.

We define functional $\mathrm{T}$ as follows:

$$
\mathrm{T}(F \cap l(\theta, \rho))=\sum_{j=1}^{m} f\left(\theta, \rho, t_{j}\right) \cdot \Delta t
$$

Let functional $\mathrm{P}$ be of the form

$$
\mathrm{P}(\mathrm{T}(F \cap l(\theta, \rho)))=\sum_{i=1}^{m} g\left(\theta_{j}, \rho_{i}\right) \cdot \Delta \rho,
$$

where $g\left(\theta_{j}, \rho_{i}\right)=\mathrm{T}\left(F \cap l\left(\theta_{j}, \rho_{i}\right)\right) ; m$ is the number of discrete values $\rho, \rho \in[-R ; R] ; R$ is the radius of the plane being scanned, i.e. radius of the recognition system retina.

We define functional $\Theta$ as follows:

$$
\Theta(P(\mathrm{~T}(F \cap l(\theta, \rho))))=\frac{\sum_{j=1}^{n} h\left(\theta_{j}\right)}{n}
$$

is the mean of functional $\mathrm{P}$, where $h\left(\theta_{j}\right)=\mathrm{P}\left(g\left(\theta_{j}, \rho\right)\right)$.

Then the feature, which is a composition of the functionals stated, is descriptive of the shaded object area within the image being studied. A wide register listing over 100 functionals and the features constructed on their basis, can be found in (Федотов, 2009).

\section{Application of Triple Features to Half-Toned Image Analysis}

The approach suggested used only to be applied to binary images, images features to be formed being their geometrical characteristics. Unlike binary images, half-tone ones possess two groups of significant characteristics: those of geometry, and brightness. Therefore, to classify halftone images, there is a good reason to design a recognition system that would take account of both geometric, and brightness characteristics of the image.

To solve the problem, two groups of triplet features were constructed:

1) those typifying geometric singularities of the image;

2) those typifying the brightness characteristics of the image.

The features of the first and the second groups possess an identical three-functional structure of the type (3). The difference between them only lies in the approach towards assigning characteristics to closely brightened segments of scanning lines. To construct features typifying geometric singularities of the image, closely brightened segments of a scanning line were put into correspondence with a geometric quantity (e.g., the length of the segment). To construct the characterizing features of the image brightness, closely brightened segments of a scanning line were put into correspondence with a certain brightness value (e.g., the average brightness of the segment). 

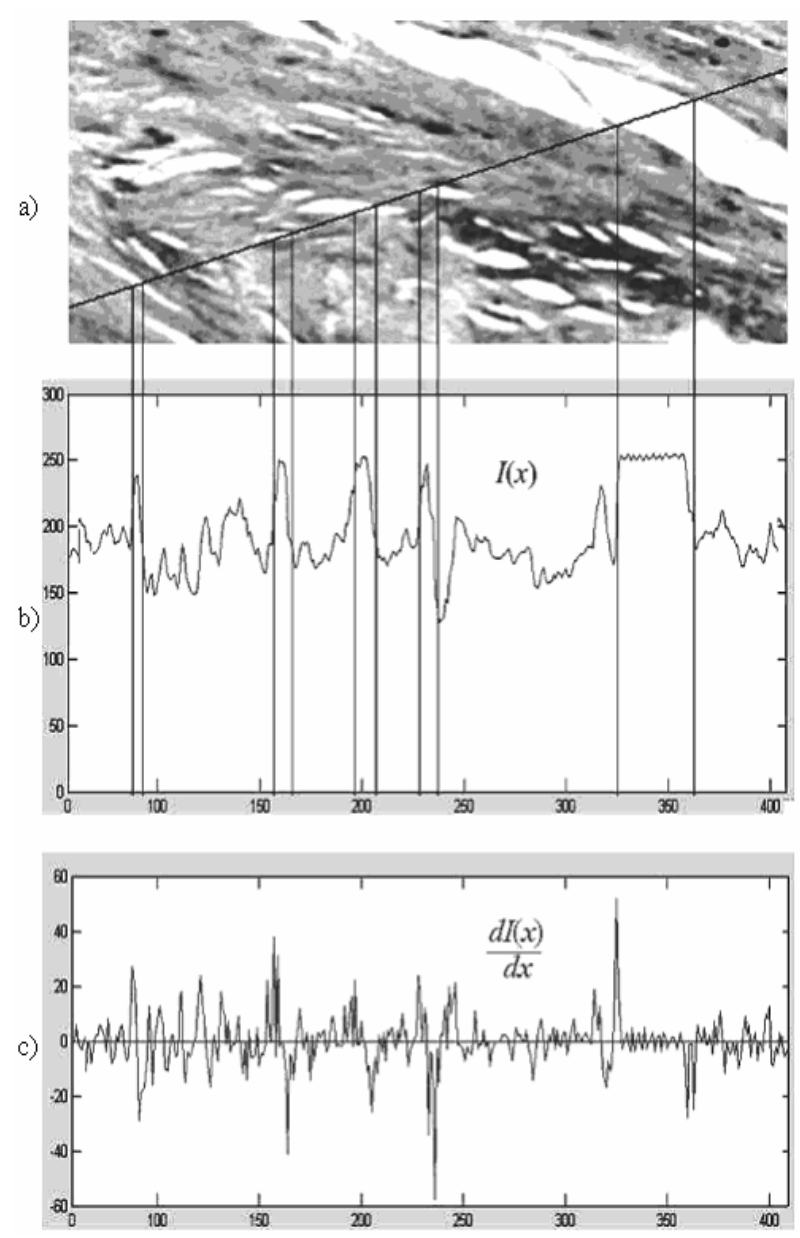

Figure 4. Image of a fibrotic tissue (a) intersected by scanning line $l(\theta, \rho)$; (b) the brightness function $I(x)$; (c) the derivative of the brightness function $\mathrm{d} l(x) / \mathrm{d} x$ along the scanning line $l(\theta, \rho)$

In Figure $4 \mathrm{~b}$, points of the scanning line are plotted against the $\mathrm{X}$-axis, and the values of the corresponding points brightness - against the $\mathrm{Y}$-axis. In Figure $4 \mathrm{c}$ points of the scanning line are plotted against the $\mathrm{X}$-axis, and the values of the derivative of the brightness function in the corresponding points - against the $\mathrm{Y}$-axis.

The boundaries of closely brightened segments of a scanning line for halftone images, are defined ambiguously, unlike the binary ones. The experiment used the following method to recognize the boundaries of closely brightened segments of a scanning line intersecting the halftone image pattern:

1) Brightness was defined at each point of the scanning line $l(\theta, \rho)$.

2) According to the data obtained, the brightness function $I(x)$ for a given line was formed.

3) The value of the brightness function derivative $\mathrm{d} I / \mathrm{d} x$ was calculated.

Its extrema helped calculate the bounce, i.e., the ajacent points of the scanning line $l(\theta, \rho)$ segments brightened closely.

The method chosen allows sufficient accuracy to determine the boundaries of homogeneous brightness of the scanning line segments, which makes it possible to find the triple recognition features theory, based on the apparatus of stochastic geometry and functional analysis, by the description of halftone images. Experiments conducted on histological images of fibrotic tissue (Федотов, Мокшанина, \& Романов, 2009) and images of cast iron microsection (Fedotov \& Mokshanina, 2010) have demonstrated the high efficiency of application of method suggested to half-tone image analysis and recognition. 


\section{Application of Triple Recognition Features to Color Textures. Feature Construction and Optimization of Evaluations}

Texture-containing images comprise those obtained through the use of airborne and satellite multispectral scanners, microscopic images of biological cells and tissue preparations, and many others. Nevertheless, in spite of textures' being prevalent and important for image recognition, there has been no universal approach towards their description and no strict definition, as yet.

A review of sources on the texture analysis problem shows that it is still beyond the capabilities of any method we know to individualize both the elements with different shades of colour, and the spatial relations between them. The existing approaches towards texture analysis use a small number of features, each of the latter generally having a narrow interpretation in terms of a specific recognition problem under work. In such cases, significant difficulties are likely to rise when extracting informative complex-structured texture features through the existing techniques.

Application of triplet recognition features to colored texture recognition has the following advantages. Firstly, it allows to automatically generate a large quantity (several thousands) of image features, Features having been constructed, the feature space has to be subject to a minimization procedure to extract the most informative features, those with the best separative power. Particularly, one of such procedures is based upon the expansion coefficients Coruna-Loeve (Fedotov, Shulga, Kol'chugin, Smol'kin, \& Romanov, 2007). Relying on a large number of features, one can allows to solve problems of recognizing complex-structured textures to be encountered in the vast majority of real images, with a sufficiently high reliability.The advantages include the fact that the method suggested enables to form features invariant to a group of motion, and to linear deformations, which proves the key to colored texture recognition. The recognition algorithm based on stochastic geometry and functional analysis is capable of individualizing both the elements with different shades of colour, and the spatial relations between them.

Trace-transformation fundamental for triple recognition feature, is a function of the image, and the scanning line intersecting it. In theoretical terms, there exist no restrictions on either texture chroma level, or the presence of object's marked boundaries.

To process color textures, the following approaches have been set up:

1) Considering a scanning line as set of concolor segments. Trace-transformation is here performed much the same as on binary images. Recognition features describe geometric singularities of distinct color areas. Criteria to isolate an area, may vary.

2) Performing trace-transformation on the scanning line histogram. The result of such a transformation describes either color, or brightness of the image, or its fragments.

3) Evaluating the color dynamics on the scanning line.

There exist two approaches to the triple recognition feature construction: the extraction, and the automatic generation. The extraction comes down to be empirical work of an expert to pick up a feature providing for a reliable characteristics of the object's singularity. The algorithm of a feature automatic generation applies a training set of objects. From all the set of triple features, a required number is to be chosen, to provide for the object most reliable recognition.

The method based on the stated approaches was tested on histological images which, in the context, were understood as colored textures in RGB model.

Histological images are obtained under the microscope within the 50- to 1000-fold magnification range, each step in magnification yielding its own fraction of test information. With a 50-fold magnification, the main object distinguished is fibrous tissue which must be dissected away for the follicles to be further treated.

Automatic generation was used to form triple features of fibrous tissue colored textures.

10340 features were obtained through automatic generation. Then, 174 ones were selected through the application of minimization procedure based on Karhunen-Loeve expansion coefficients. The decision function described in detail in (Fedotov \& Mokshanina, 2010), was built up with provision for the weighting factor of each informational feature.

The experiment described demonstrated that the minimum value of the classification mean error for a group of informational features makes 3,7 percent. The result obtained implies sufficiently high efficiency of the triple-feature theory application to the problem of analyzing fibrous tissue colored textures. 
Triplet recognition feature calculation involves a lot of computational effort, but the algorithm allows for a highly efficient use of parallel processing. Triplet features of color textures recognition provide for algorithm optimization capabilities enhancing, and can, therefore, efficiently use the up-to-date computer resources.

Color texture processing is a promising trend of pattern recognition theory development. The work is by no means to be considered complete, but the experiments have demonstrated that triplet features of recognition can be efficiently used for color texture processing. Further developments involve large-scale experiments for the analysis and recognition of colored textures in the field of biology.

\section{Discussion}

There is a broad class of technical and medical diagnostics problems where the key information resides in visual images. In our case, we studied the problem of analysis and recognition of half-tone images and color textures. To solve the problem, we proposed a new approach based on stochastic geometry that helped create a new class of recognition design features - the triplet ones. A key element to their formation is the image new geometric transformation (a Trace-transform) involving image scanning along complex trajectories. The features' singularity is that they are structured as a composition of three functionals. With that structure, we can generate a a mass (tens of thousands) of features, which enables to enhance recognition flexibility, versatility, and reliability.

Moreover, it has been established that the theory based on triple features yields a stable result when analyzing biological micro- and nano- objects, in particular, when recognizing images in the field of nanotechnology. Results have been obtained in a successful recognition of half-tone histological images of fibrotic tissue (see Fedotov \& Shulga, 2004). Beyond that, the theory of triple features stated above has been tested on and proved suitable for technical diagnostics and flaw detection problems. The concept applicability has been experimentally proved in (Fedotov \& Mokshanina, 2010). The experiments have demonstrated that triplet features of recognition can be effectively used for color texture processing. Further plans call for the extension of the trace-transform and the triple features theory to the tasks of 3D images analysis and recognition.

The theory constructed helps to obtain features independent of object motions or linear deformation, i.e. affine transformations. Furthermore, we can apply the theory to obtain features which are sensitive to the above mentioned transformations, giving us a possibility to compute the parameters of objects' affine transformations (that could be helpful for a machine vision system to position a robotic tools precisely).

\section{Acknowledgements}

Work was performed with financial support the Russian fund of fundamental researches, the project № 12-07-00501.

\section{References}

Федотов, Н. Г. (1990). Методы стохастической геометрии в распознавании образов. Москва: Радио и связь. (Fedotov, N.G. (1990). Methods of Stochastic Geometry in Patters Recognition. Moscow: Radio and Communications).

Федотов, Н. Г. (2009). Теория признаков распознавания образов на основе стохастической геометрии и функиионального анализа. Москва: ФИЗМАТЛИТ. 304 с. (Fedotov N.G. (2009) Theory for Image Recognition Features Based on Stochastic Geometry and Functional Analysis. Moscow: Fizmatlit.)

Kendall, W. S., \& Molchanov, I. (2010). New Perspectives in Stochastic Geometry. Oxford, UK: Oxford University Press.

Kadyrov, A. A., Saveleva, M. V., \& Fedotov, N. G. (1994). Image Scanning Leads to Alternative Understanding of Image. Third Int. Conf. on Automation, Robotics and Computer Vision (ICARCV'94), Singapore.

Fedotov, N. G., \& Kadyrov, A. A. (1995). Image Scanning in Machine Vision Leads to New Understanding of Image. In Proc. of 5th International Workshop on Digital in Processing and Computer Graphics, Proc. International Society for Optical Engineering (SPIE), Vol. 2363, pp. 256-261.

Fedotov, N. G., \& Shulga, L. A. (2000). New Theory of Pattern Recognition Feature on the Basis of Stochastic Geometriy. WSCG'2000 Conference Proceedings, University of West Bohemia, 1(2), 373-380.

Kadyrov, A. A., \& Fedotov, N. G. (1995). Triple Features Pattern Recognition and Image Analysis. Advances in Mathematical Theory and Applications, 5(4), 546-556.

Федотов, Н. Г., Кольчугин, А. С., Смолькин, О. А., Моисеев, А. В., \& Романов, С. В. (2008). Формирование признаков распознавания сложноструктурированных изображений на основе 
стохастической геометрии. Измерительная техника. № 2. С. 56-61 (с 1958 г. переводится в США под названием Measurement Techniques) (Fedotov, N.G., Kol'chugin, A.S., Smol'kin, O.A., Moiseev, A.V. (2008). Feature Generation for Recognition of Complex-structured Images Based on Stochastic Geometry. Measurement Techniques. No 2. pp. 56-61)

Vidal, M., \& Amigo, J. M. (2012). Pre-processing of Hyperspectral Images. Essential Steps Before Image Analysis. Chemometrics and Intelligent Laboratory Systems, 117(1), 138-148. http://dx.doi.org/10.1016/j.chemolab.2012.05.009

Федотов, Н. Г., \& Никифорова, Т. В. (2002). Техническая дефектоскопия на основе новой теории распознавания образов. Измерительная техника. № 12. C. 27-31 (Fedotov, N.G., Nikiforova, T.V. (2002). Technical Defectoscopy Based on New Image Recognition Theory. Measurement Techniques. No 12. pp. 27-31).

Fedotov, N. G., Shulga, L. A., \& Roy, A. V. (2004). Visual Mining for Biomatrical System Based on Stochastic Geometry. Proc. Int. Conf. Pattern Recognition and Image Analysis. PRIA-7-2004, Vol. 2, pp. 473-475.

Shin, B. S., Cha, E. Y., Kim, K. B., Cho, K. W., Klette, R., \& Young, W. W. (2008). Effective Feature Extraction by Trace Transform for Insect Footprint Recognition. 3rd International Conference on Bio-Inspired Computing: Theories and Applications, BICTA 2008 sponsors: IEEE South Australia Section. Adelaide, NT. pp. 97-102.

Fooprateepsiri, R., \& Kurutach, W. (2010). Highly Robust Approach Face Recognition Using Hamming - Trace Combination. Proc. of the IADIS Int. Conf. Intelligent Systems and Agents 2010, Proc. of the IADIS European Conference on Data Mining 2010, Part of the MCCSIS 2010. pp. 83-90

Fedotov, N. G., Shulga, L. A., Kol'chugin, A. S., Smol'kin, O. A., \& Romanov, S. V. (2007). Triple Features of Ultrasonic Image Recognition. Proc. Of the 8th Int. Conf. on Pattern Recognition and Image Analysis (PRIA-8-2007). Yoshkar-Ola, Russia. Vol.1. pp. 299-300.

Fedotov, N. G., \& Shulga, L. A., (2004). Feature Generation and Stochastic Geometry. In Proc. of the 4th International Workshop on Pattern Recognition in Information Systems, PRIS'2004. Porto, Portugal, pp. $169-175$.

Федотов, Н. Г., Мокшанина, Д. А., \& Романов, С. В. (2009). Анализ текстур гистологических изображений. Математические методы распознавания образов (ММРО-14): труды Всероссийской конференции. Москва: МАКС Пресс. С. 611-613. (Fedotov N. G., Mokshanina D. A., Romanov S. V., 2009. Analysis of Textures of Histological Images. Proc. Of the $14^{\text {th }}$ Int. Conf. Mathematical Methods of Pattern Recognition (MMRO-14). Suzdal, Russia. Moscow: MAKS Press. pp. 611-613.)

Fedotov, N. G., \& Mokshanina, D. A. (2010). Recognition of Halftone Textures from the Standpoint of Stochastic Geometry and Functional Analysis. Pattern Recognition and Image Analysis, 20(4), 551-556. http://dx.doi.org/10.1134/S1054661810040164

\section{Copyrights}

Copyright for this article is retained by the author(s), with first publication rights granted to the journal.

This is an open-access article distributed under the terms and conditions of the Creative Commons Attribution license (http://creativecommons.org/licenses/by/3.0/). 